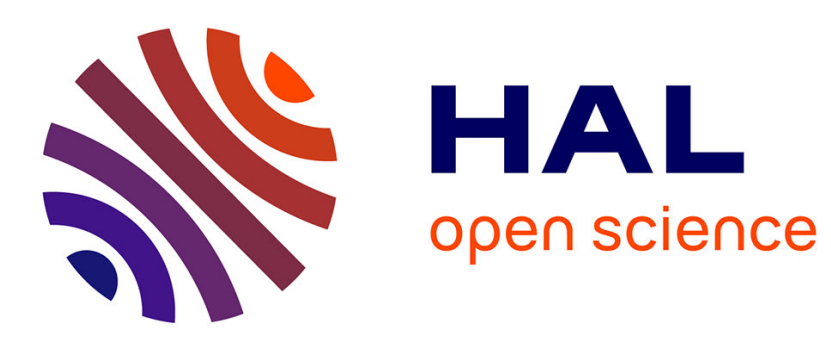

\title{
Description d'une cible gazeuse pour étude des réactions nucléaires
}

\author{
J.P. Cremet, M. Lambert, G. Lefevre, L. Rais
}

\section{To cite this version:}

J.P. Cremet, M. Lambert, G. Lefevre, L. Rais. Description d'une cible gazeuse pour étude des réactions nucléaires. Revue de Physique Appliquée, 1966, 1 (4), pp.267-272. 10.1051/rphysap:0196600104026700 . jpa-00242732

\section{HAL Id: jpa-00242732 https://hal.science/jpa-00242732}

Submitted on 1 Jan 1966

HAL is a multi-disciplinary open access archive for the deposit and dissemination of scientific research documents, whether they are published or not. The documents may come from teaching and research institutions in France or abroad, or from public or private research centers.
L'archive ouverte pluridisciplinaire HAL, est destinée au dépôt et à la diffusion de documents scientifiques de niveau recherche, publiés ou non, émanant des établissements d'enseignement et de recherche français ou étrangers, des laboratoires publics ou privés. 


\title{
DESCRIPTION D'UNE CIBLE GAZEUSE POUR ÉTUDE DES REACTIONS NUCLÉAIRES
}

\author{
Par J. P. CREMET, M. LAMBERT, G. LEFEVRE et L. RAIS, \\ Institut d'Études Nucléaires d'Alger.
}

\begin{abstract}
Résumé. - On décrit une chambre à cible gazeuse munie d'un dispositif de pompage différentiel et destinée à étudier des réactions nucléaires. L’appareillage électronique annexe est aussi décrit et des résultats expérimentaux typiques sont présentés.
\end{abstract}

\begin{abstract}
For studying nuclear reactions, a gaseous target assembly using a differential pumping unit is described.

The electronic detection equipment is describedand typical experimental results are presented.
\end{abstract}

Introduction. - Dans l'étude des réactions nucléaires provoquées par un faisceau incident sur un élément cible, il y a de très nombreux avantages à réaliser cette cible sous forme gazeuse. Une telle cible est en effet sans support, et par suite, préservée des diffusions parasites nucléaires ou coulombiennes créées par celui-ci. Son épaisseur, fonction de la pression du gaz étudié, est continuement variable et aussi faible que l'on veut, et son homogénéité est parfaite. Le nombre de noyaux contenus dans la cible peut-être déterminé avec précision en mesurant volume, pression et température. Des mesures absolues sont possibles. Une cible gazeuse peut être bombardée par des faisceaux intenses sans subir de détériorations. Aucun dépôt superficiel, de carbone par exemple, n'introduit de "straggling " ou de contamination comme dans le cas d'une cible solide. Enfin, une excellente mesure du " bruit de fond " de l'appareillage expérimental est aisément réalisée en observant les spectres de diffusion enregistrés par les détecteurs sur la chambre vide.

Beaucoup d'éléments naturellement gazeux sont isotopiquement presque purs. Enfin de nombreux éléments peuvent être rendus gazeux, par exemple sous la forme $\mathrm{SH}^{2}, \mathrm{PH}^{3}, \mathrm{SiH}^{4}, \mathrm{CH}^{4}$.

L'hydrogène joue alors le rôle d'un support très léger. La diffusion parasite sur l'hydrogène est alors limitée dans le système du laboratoire à des angles de diffusion toujours inférieurs à $90^{\circ}$ et les masses atomiques de la cible et de l'hydrogène sont si différentes qu'il est très aisé de séparer leurs produits de réaction.

1. L'appareillage mécanique. - 1-1. Description DE LA CHAMBRE DE RÉACTION. - Nous pouvons définir, à l'intérieur de cette chambre qui contient



Fig. 1. - Chambre pour cibles gazeuses. 
le gaz étudié, une région active intérieure au cylindre contenant le faisceau et une région observée intérieure au cône définissant l'angle solide dans lequel les particules secondaires émises sont observées par les détecteurs. La cible gazeuse est la région commune aux deux précédentes. Il en résulte que les cônes d'observation et le cylindre contenant le faisceau doivent être définis géométriquement donc mécaniquement avec une précision maximum.

La chambre est essentiellement constituée d'un cvlindre en laiton de $200 \mathrm{~mm}$ de diamètre intérieur et de $112 \mathrm{~mm}$ de hauteur, fermé supérieurement par un couvercle mobile tournant sous vide, sur un joint en néoprène et dont la position est repérée par un vernier. Un détecteur fixe qui joue le rôle de moniteur dans les distributions angulaires est placé sur le corps de la chambre et fait un angle de $165^{\circ}$ avec la direction du faisceau. Douze ensembles de détection des particules diffusées, constitués d'une jonction $\mathrm{L}$ et éventuellement d'une jonction par transmission $\mathrm{K}$, inclinés chacun de $21^{\circ} 30^{\prime}$ par rapport au plan horizontal, sont fixés sur le couvercle mobile qui peut tourner complètement autour de l'axe vertical de la chambre en glissant sur un joint torique. L'angle solide de l'observation de chaque détecteur défini par le diaphragme $H$ est de $3,7^{\times 10^{-3}}$ stéradient pour une jonction de $80 \mathrm{~mm}^{2}$ de surface de détection. L'angle d'ouverture et, par suite la dispersion angulaire maximum, est de 4 degrés. L'ensemble des deux diaphragmes $\mathrm{H}$ et $\mathrm{J}$ détermine la longueur de la cible gazeuse observée, à laquelle est liée une nouvelle dispersion angulaire inférieure à 7 degrés. Un cristal (INa) M et un ensemble de détection des $\gamma$ peuvent être placés sur le couvercle. Le gaz est introduit dans la chambre par un robinet à fuite réglable et sa pression est mesurée par un manomètre à huile $\mathrm{APIEZON} B$ et un capteur ECA dont la mesure est transmise à distance.

La pression de la cible est toujours supérieure à $0,5 \mathrm{~mm}$ de $\mathrm{Hg}$. Une valeur plus faible réduit le taux de production des particules secondaires émises sans aucun gain appréciable sur la résolution en énergie des particules primaires et secondaires de la réaction, celle-ci ne pouvant être supérieure à celle donnée par l'accélérateur. Par contre, la pression ne peut excéder $2 \mathrm{~cm}$ de $\mathrm{Hg}$ sans que cette résolution ne soit très dégradée par le "straggling " produit dans le gaz.

1-2. Le pompage différentiel. -- Au lieu de séparer la chambre gazeuse de l'enceinte à vide de l'accélérateur au moyen d'une feuille mince qui introduit nécessairement un "straggling " supplémentaire, un dispositif de pompage différentiel a été réalisé. Cette solution consiste à interposer entre la chambre de diffusion de pression $P_{1}$

$$
\left(0,5 \mathrm{~mm} \mathrm{Hg}<\mathrm{P}_{1}<20 \mathrm{~mm} \mathrm{Hg}\right)
$$

et l'enceinte à vide de l'accélérateur de pression $P_{4}$ $\left(P_{4} \leqslant 10^{-5} \mathrm{~mm} \mathrm{Hg}\right)$ deux autres chambres intermédiaires à des pressions intermédiaires $P_{2}$ et $P_{3}$ telles que $P_{1} \gg P_{2} \gg P_{3} \gg P_{4}$. La chute de pression entre deux chambres successives est obtenue au moyen d'un ou de plusieurs diaphragmes séparant ces deux chambres, à la traversée desquels le gaz qui s'écoule continuement subit cette brusque chute de pression. Pour maintenir les pressions constantes dans chacune des chambres et obtenir ainsi un équilibre dynamique, on pompe en permanence dans chacune des chambres intermédiaires.

Un premier diaphragme $\mathrm{C}$ d'un diamètre de 1,25 mm sépare la chambre de réaction d'une deuxième chambre où une pompe $B$ d'un débit de $12,5 \mathrm{l} / \mathrm{sec}$ maintient une pression inférieure à $5 \times 10^{-2} \mathrm{~mm} \mathrm{Hg}$. Un ensemble $\mathrm{A}$ de 15 diaphragmes d'un diamètre de $1,5 \mathrm{~mm}$ sépare cette deuxième chambre d'une troisième et d'une quatrième où des pompes moléculaires maintiennent des vides meilleurs que $10^{-4} \mathrm{~mm} \mathrm{Hg}$ et $10^{-5} \mathrm{~mm} \mathrm{Hg}$. Cet ensemble de diaphragmes, en tantale, pour minimiser le bruit de fond est réparti sur une longueur de un mètre environ et définit la section du faisceau en réduisant sa dispersion angulaire à \pm 5 minutes d'angle.

Du point de vue de la dispersion qu'il provoque sur l'énergie des protons, le dispositif de pompage différentiel est équivalent à une feuille mince d'une épaisseur de 0,1 keV dans le cas d'une cible d'argon et pour des protons d'une énergie de $1 \mathrm{MeV}$.

1-3. Le Collecteur DE COURANT DE FAISCEAU. Le faisceau est focalisé de telle sorte que son intensité moyenne au niveau de la cible est de $0,8 \mu \mathrm{A}$.

L'intensité maximum admise par la feuille de nickel désignée ci-dessous, sans détérioration est de 1,2 $\mu \mathrm{A}$. Cette intensité est mesurée par un intégrateur de courant relié à un collecteur F en tantale sur lequel le faisceau vient tomber. Ce collecteur est séparé de la chambre par une feuille de nickel D de $0,8 \times 10^{-3} \mathrm{~mm}$ ou $1,3 \times 10^{-3} \mathrm{~mm}$ d'épaisseur et une pompe moléculaire $G$ y maintient un vide meilleur que $10^{-5} \mathrm{~mm} \mathrm{Hg}$. Un anneau $\mathrm{E}$ de garde porté à - 300 volts repousse les électrons secondaires et un diaphragme l'empêche d'être atteint par le faisceau diffusé par la feuille de nickel. Celle-ci n'est distante que de $6 \mathrm{~cm}$ du centre de la chambre, et la pression de la cible est suffisamment faible pour que l'intensité du faisceau diff.usé par le gaz dans la chambre soit négligeable.

L'ensemble constitué de la chambre de réaction et des éléments annexes est placé sur le banc d'une fraiseuse de facon à venir placer sans difficulté l'axe défini par les diaphragmes du pompage différentiel en coïncidence avec la direction du faisceau.

2. L'appareillage électronique. - Le rôle de l'électronique est de permettre l'analyse simultanée de 8 


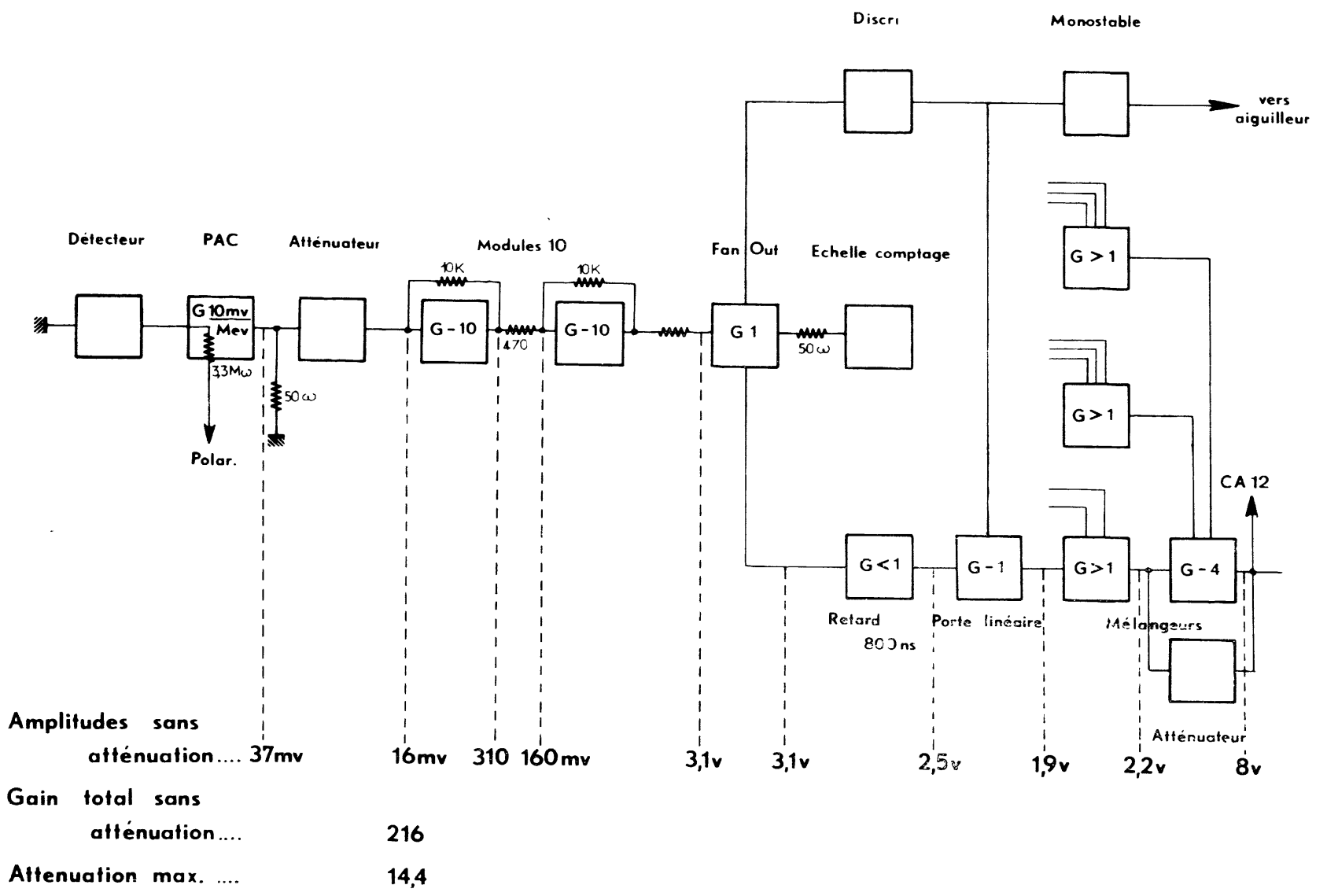

FIG. 2.

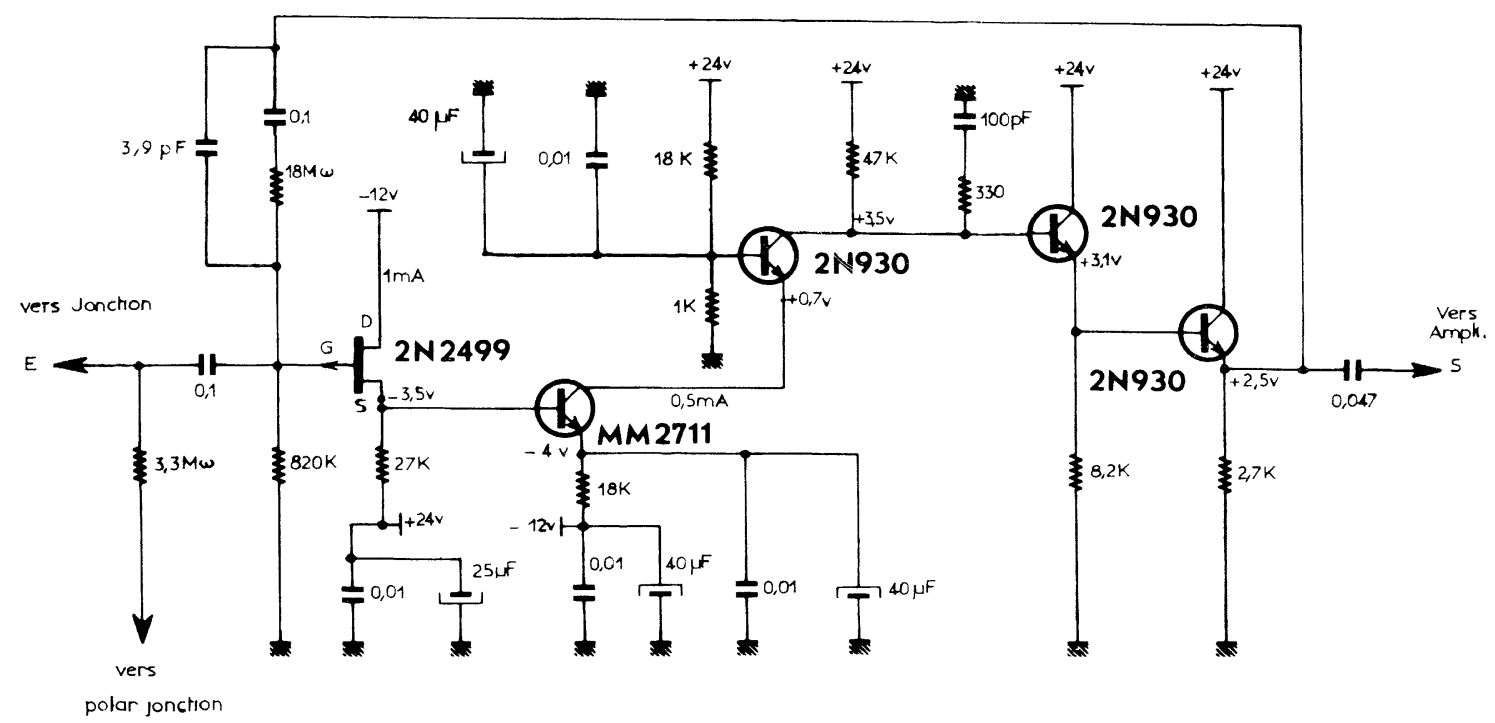

FIg. 3.

ou 12 voies de spectrométrie avec un ensemble Intertechnique, convertisseur analogique-digital CA 12, bloc-mémoire BM 48, aiguilleur AP 32.
2-1. Les préamplificateurs. - Les détecteurs des particules chargées sont des jonctions dont le signal est amplifié par un préamplificateur de 
charges qui, pour jouer son rôle, doit avoir une impédance d'entrée en boucle ouverte beaucoup plus grande que l'impédance de contre-réaction, un gain en tension en boucle fermée, négatif, élevé. Ces conditions étant remplies, la capacité de contreréaction est alors choisie arbitrairement petite. Nous avons pris $C=3,9 \mathrm{pF}$, c'est-à-dire que, la jonction donnant 4,8 $\times 10^{-14}$ coulomb/MeV, le préampli donnera des impulsions d'amplitude

$V_{\mathbf{s}}=4,8 \times 10^{-14} / 3,9 \times 10^{-12}=12 \mathrm{mV} / \mathrm{MeV}$,

ce qui fait à l'entrée du préampli $30 \mu \mathrm{V} / \mathrm{MeV}$.

La réalisation du préampli a été guidée par le souci d'obtenir une grande capacité dynamique (ce qui le rend utilisable avec des jonctions de grande surface ou peu profonde), et une bonne résolution, résultat inhérent à un faible bruit, et à une bonne stabilité de la capacité de contre-réaction, l'amplitude des impulsions de sortie étant $Q / C(Q$ : charges créées dans la jonction, $C$ : capacité de contreréaction).

Le transistor 2N2499, à effet de champ, présente l'avantage d'avoir une grande impédance d'entrée et un faible bruit. L'ensemble cascode, ayant un gain de puissance élevé, donne un bon rapport signal sur bruit.

L'ensemble est contre-réactionné par une résistance de $18 \mathrm{M} \omega$ qui stabilise le gain, diminue le bruit du préampli, et fixe la constante de temps de décharge de la capacité d'entrée, constante de temps choisie suffisamment longue pour qu'il y ait totale collection des charges de la jonction et pour que les liaisons capacitives de toute la chaîne qui suivra ne provoquent pas d'overschoot qui pourraient être gênants (empilement sur des overshoot mal restitués).

Résumé des caractéristiques du préampli :

- Capacité dynamique. $1600 \mathrm{pF}$.

- Gain en tension.... - 400 .

- Signe des impulsions

admises .......... + ou -.

- Amplitude maximum

de sortie ........ 3 volt.

- Bruit propre au préampli .............

Indécelable à l'oscilloscope (sensibilité $5 \mathrm{mV} /$ $\mathrm{cm})$.

2-2. Les amplificateurs. - 2-2-1. Les amplificateurs de gain-10. - C'est un système qui a une grande impédance d'entrée en boucle ouverte (le premier transistor est monté en émetteur suiveur), un grand gain négatif en boucle fermée, et qui est contre-réactionné par une résistance de valeur peu élevée, ce qui lui donne une faible impédance d'entré en boucle fermée.

Le gain en boucle fermée est de 500 . La résistance de contre-réaction étant $10 \mathrm{k} \Omega$, l'impédance d'entrée est alors de $20 \mathrm{ohms}$.

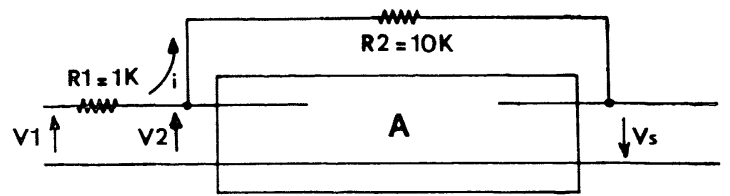

Fig. 4.

Le module amplificateur a ainsi un gain en tension

$$
V_{\mathrm{s}} / V_{1} \# R_{2} / R_{1}=-10 \text {. }
$$

Cet ampli admet indifféremment des signaux positifs ou négatifs, d'amplitude 10 volts.

Les transistors utilisés sont à grand gain et faible bruit ; cela, ajouté à une forte contre-réaction fait de ce module un ampli à faible bruit, propre à amplifier les faibles signaux du préampli.

2-2-2. La patte d'oie. - Cet élément sert à envoyer le même signal sur plusieurs circuits, sans que les fonctionnements respectifs de ces derniers soient altérés.

Il consiste en une cascade de transistors montés collecteurs communs, leurs bases étant reliées. Ils sont polarisés de façon que le gain de courant soit maximum, et la résistance d'émetteur est choisie grande pour que l'on obtienne un gain en tension voisin de l'unité et une faible impédance de sortie.

2-2-3. Les mélangeurs. - Principe d'un mélangeur : c'est un circuit d'impédance d'entrée aussi faible que possible; De cette façon, chaque voie du mélangeur ne "voit " pas les autres.

Dans le mélangeur de gain, + 1, l'impédance d'entrée est celle d'un transistor base à la masse. Il est suivi de deux transistors collecteur commun.

Le gain est $\mathrm{Rh}_{21} \mathrm{i} / \mathrm{rh}_{21} \mathrm{i}=R / r$.

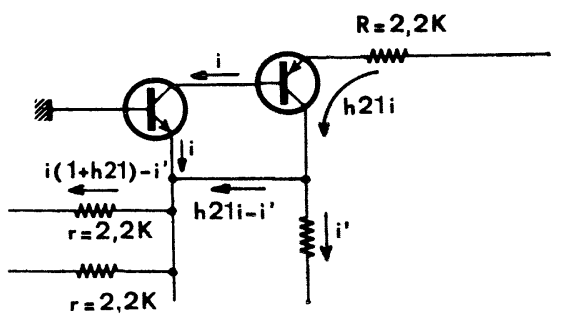

FIG. 5.

S'il arrive une impulsion simultanément sur les deux voies, toutes choses égales par ailleurs, on a alors dans chaque résistance $r$ un courant $i / 2$. Le gain est alors $2 R / r$, c'est-à-dire que les limpulsions s'additionnent algébriquement.

Les impulsions admises sont négatives d'amplitude maximum $10 \mathrm{~V}$. Le mélangeur de gain - $\mathbf{4}$ est un module de gain 10 modifié. Ce circuit a une faible impédance d'entrée due à la contre-réaction. On a mis sur l'entrée trois résistances de $2,2 \mathrm{k} \Omega$ pour en faire un mélangeur.

2-2-4. La porte linéaire. - Cette plaquette comprend l'ensemble porte linéaire et le monostable d'ouverture de la porte. 


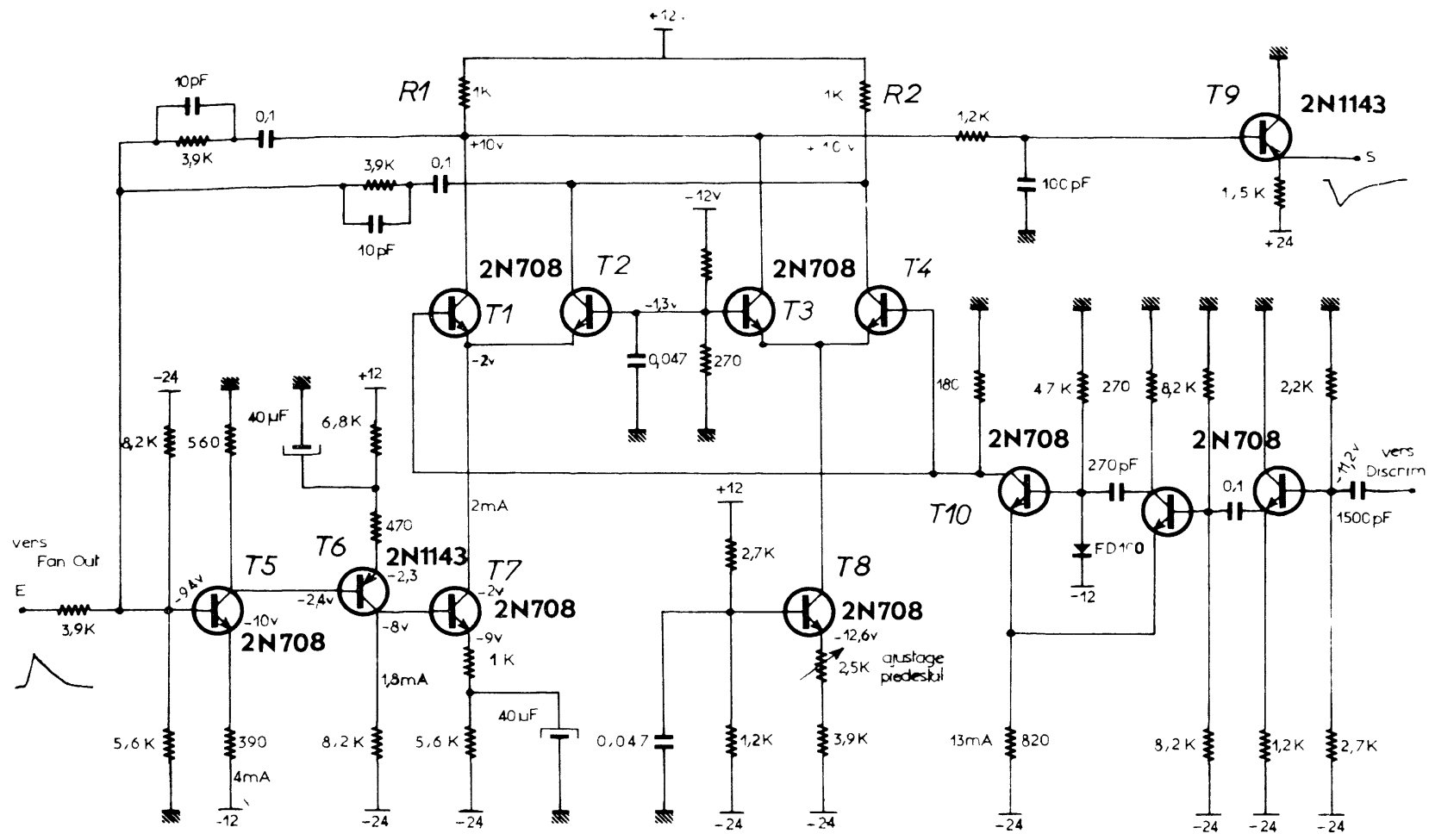

FIG. 6.

La porte linéaire est constituée d'un ensemble amplificateur T5, T6, T7, d'un transistor T8 qui délivre un courant constant, d'un ensemble porte T1, T2, T3, T4, d'un étage de sortie T9.

Les polarisations sont telles que $\mathrm{T} 2$ et $\mathrm{T} 3$ sont conducteurs, T4 et T1 étant bloqués. T1 étant bloqué, tout signal arrivant sur son émetteur n'ira pas au-delà, et ne sera pas vu sur la charge de $1 \mathrm{~K}$. Le courant de polarisation de T2 est celui 'de T7, et T3 est polarisé par T8. Quand le monostable de temps, recevant une impulsion du discriminateur, bascule, T10 se ferme. Son collecteur remonte au potentiel de masse. Alors $\mathrm{T} 4$ et $\mathrm{T} 1$ conduisent, faisant remonter les potentiels collecteurs de T7 et T8, de -2 à $-0,7$ volt, ce qui bloque T2 et T3.

Le courant qui passait dans T3 et R1 passe alors dans T4 et R2 ; celui qui passait dans T2 et R2 passe alors dans T1 et R1. Si ces courants sont rigoureusement identiques, il n'y aura aucune variation de potentiel sur R1 ; d'où la nécessité d'ajuster l'un des courants par rapport à l'autre, de façon à annuler tout piédestal sur R1. Le transistor T1 étant ouvert, les impulsions de T7 sont alors transmises par T1 à T9.

A l'ouverture et à la fermeture de la porte, cette fermeture se produisant dans le monostable de temps bascule à nouveau à son état initial, il se produit, notamment sur R1, un signal parasite (charge ou décharge de capacités parasites, commutations non simultanées des transistors). Ce signal parasite a été réduit en découplant les points de potentiels fixes (bases de T2, T3, T8) pour empêcher les fluctuations des courants de polarisation, et en intégrant le signal parasite en amont de T9. Finalement, en sortie de T9, son amplitude est inférieure à celle du plus petit signal à analyser, son temps de montée court (15 ns) l'empêchant de surcroît d'être analysé par le CA 12.

Cette porte a été réalisée pour empêcher le passage du bruit de fond. Elle remplit parfaitement son rôle, le signal passant, quand la porte est fermée, étant atténué d'un facteur 100.

3. Résultats expérimentaux. - Afin d'illustrer les résultats expérimentaux qui peuvent être obtenus avec l'appareillage qui vient d'être décrit, la figure 7 indique le spectre des particules secondaires émises vers l'arrière, pour $\Theta_{\mathrm{lab}}=165^{\circ}$ par une cible d'air bombardée par des deutons de $2 \mathrm{MeV}$ d'énergie.

Ce spectre a été tracé en deux fois, un premier spectre non dilaté permettant d'observer sur 256 canaux les groupes de particules bien séparés de grande énergie, comprenant les groupes $\alpha_{0}(9,914 \mathrm{MeV}), \mathrm{p}_{0}, \alpha_{1}$ et $\alpha_{2}(4,651 \mathrm{MeV})$ et un deuxième spectre dilaté, obtenu en doublant le gain des chaînes électroniques, permettant d'observer sur 512 canaux les particules d'énergie plus faible. Ces deux spectres sont accolés sur la figure 7. Les partj- 


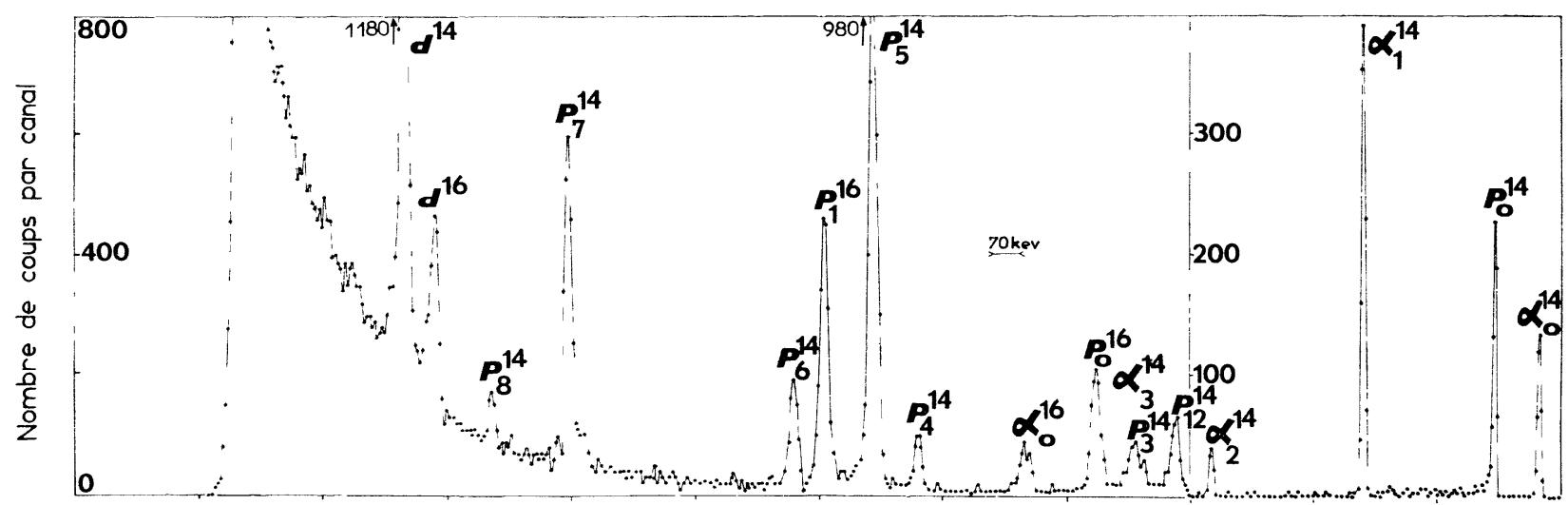

Fig. 7. - Spectre des produits des réactions induites sur l'air par des deutons de $2 \mathrm{MeV}$ à wW $=165^{\circ}$.

cules correspondant à la cible d'azote 14 portent l'indice supérieur 14, celles correspondant à l'oxygène 16, l'indice 16. On observe nettement les groupes $\alpha_{0}, \alpha_{1}, \alpha_{2}$, et $\alpha_{3} ; p_{0}, p_{3}, p_{4}, p_{5}, p_{6}, p_{7}$ et $p_{8}$ sur ${ }^{14} \mathrm{~N}$. Les groupes $p_{1}$ et $p_{2}$ sont séparés par $27 \mathrm{keV}$ d'énergie et commencent à être séparés à certains angles. Le doublet correspondant n'apparaît pas sur la figure 2 car pour $\Theta_{\text {lab }}=165^{\circ}$ et pour $E_{\text {lab }}=2^{\prime} \mathrm{MeV}$, on sait que la section efficace relative au groupe $p_{2}$ est près de quatre fois plus faible que celle du groupe $\mathrm{p}_{1}$. D'ailleurs l'épaulement correspondant est visible sur la figure 7 . On observe les groupes $\alpha_{0}$, $p_{0}$ et $p_{1}$ sur ${ }^{16} \mathrm{O}$. L'écart en énergie entre les deux groupes $p_{1}^{16}$ et $p_{6}^{14}$, totalement séparés est de $80 \mathrm{keV}$. La pression de la cible était de $4 \mathrm{~mm} \mathrm{Hg}$ et l'intensité du faisceau incident égal à $0,8 \mu \mathrm{A}$. La remontée observée dans la partie basse du spectre est essen- tiellement due aux neutrons créés par les réactions parasites (dd).

Il y a tout particulièrement lieu de remarquer que ce spectre a été enregistré en même temps que les autres spectres relatifs aux autres voies. La résolution obtenue (largeur à mi-hauteur du pic $p_{5}^{14}$ inférieure à $28 \mathrm{keV}$ ) est à peu près celle de la jonction ORTEC utilisée et celle que l'on obtient en stockant les particules émises dans une seule voie à la fois. Ce dernier résultat montre que les portes linéaires remplissent parfaitement leur rôle. Enfin la résolution globale obtenue et l'inexistence de groupe de particules parasites confirme l'intérêt d'une cible gazeuse équipée d'un dispositif de pompage différentiel.

Manuscrit reçu le 10 juin 1966. 